165. Fritz Bethmann, Ueber o-Xylalphtalid.

(Aus dem I. Berl. Univ.-Laborat.)

[Eingegangen am 10. April.]

Im Anschluss an die Arbeiten von E. Heilmann ${ }^{1}$ ) resp. A. Ruhemann ${ }^{2}$ ) über $m$ - resp. $p$-Xylalphtalid habe ich die entsprechende $o-V$ erbindung dargestellt und eingehend untersucht.

Die zu meinen Versuchen erforderliche o-Tolylessigsäure $\mathrm{CH}_{3} \cdot \mathrm{C}_{6} \mathrm{H}_{4} \cdot \mathrm{CH}_{2} \cdot \mathrm{CO}_{2} \mathrm{H}^{3}$ ) konnte ich in einer Ausbeute von $67 \mathrm{~g}$ (d. h. $47 \mathrm{pCt}$. der Theorie) aus $100 \mathrm{~g} \mathrm{o-Xylol} \mathrm{gewinnen,} \mathrm{welches} \mathrm{im} \mathrm{Sonnen-}$ licht bromirt, mit Cyankalium umgesetzt und dann ohue Isolirung des Amids (s. 1. c.) mit Salzsäure im Rohr bei $100^{\circ}$ verseift wurde.

\title{
Darstellung des $0-\mathrm{Xylalphtalids.}$
}

Man erhitzt gleiche Gewichtstheile o-Tolylessigsäure und Phtalsäureanhydrid mit 1/100 des Gesammtgewichtes Natriumacetat im Kolben 10 Stunden lang auf $240-250^{\circ}$, wobei eine gleichmässige Gasentwickelung erfolgt. Die nach dem Erkalten erstarrte Masse krystallisirt aus siedendem absolutem Alkohol in gelben Schuppen vom Schmp. $136.5^{\circ}$; welche aus

bestehen.

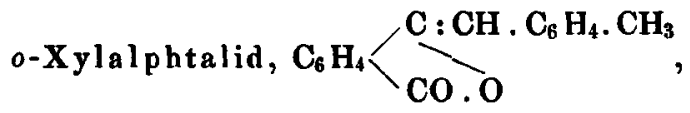

Die Ausbeute beträgt $80 \mathrm{pCt}$. der Theorie. Es ist unvortheilhaft, den Versuch mit mehr, als etwa $15-20 \mathrm{~g}$ anzustellen, da sonst die Ausbeute vermindert wird.

Der Körper ist in Wasser nicht, in Ligroïn wenig, besser in warmem Methyl-, Aethyl-; Anyl-Alkohol, Aether, Schwefelkohlenstoff nnd Eisessig, leicht in Benzol, Chloroform und Essigester löslich.

0.1685 g Sbst.: $0.5015 \mathrm{~g} \mathrm{CO}_{2}, 0.0813 \mathrm{~g} \mathrm{H}_{2} \mathrm{O}$.

$$
\begin{array}{r}
\mathrm{C}_{16} \mathrm{H}_{12} \mathrm{O}_{2} \text {. Ber. C 81.36, H 5.10. } \\
\text { Gef. " 81.17, 》5.36. }
\end{array}
$$

Durch Erhitzen mit Kalilauge verwandelt sich der Körper unter W asseraufnahme in

$$
\begin{gathered}
\left(\ell-o-M e t b y l-d \text { esoxybenzoïn-o-carbonsäure }{ }^{4}\right), \\
\mathrm{CO}_{2} \mathrm{H} \cdot \mathrm{C}_{6} \mathrm{H}_{4} \cdot \mathrm{CO} \cdot \mathrm{CH}_{2} \cdot \mathrm{C}_{6} \mathrm{H}_{4} \cdot \mathrm{CH}_{3},
\end{gathered}
$$

welche mit Salzsäure als teigige, bald erstarrende Masse ausfällt, aus. siedendem Wasser (ca. 230 Th.) umkrystallisirt, in feinen, weissen

b) Diese Berichte 23, $3159 . \quad$ 2) ebenda 24, 3964.

3) Radziszewski und Wispek, diese Berichte 15, 1747; 18, 1281; J. Schramm, ebenda 18, 1278.

4) "a " zum Unterschiede von einer weiter unten bescbriebenen isomeren Sarure, die $\gg \beta \ll$ genannt wird. 
Nadeln, vom Schmp. $131^{\circ}$ anschiesst und in den üblichen Mitteln leicht löslich ist.

0.1790 g Sbst.: $0.4961 \mathrm{~g} \mathrm{CO}_{2}, 0.0948 \mathrm{~g} \mathrm{H}_{2} \mathrm{O}$.

$0.1901 \mathrm{~g}$ Sbst. : $0.5269 \mathrm{~g} \mathrm{CO}_{2}, 0.0996 \mathrm{~g} \mathrm{H}_{2} \mathrm{O}$.

$$
\begin{array}{ll}
\mathrm{C}_{16} \mathrm{H}_{14} \mathrm{O}_{3} \text {. } & \text { Ber. C 75.59, H 5.52. } \\
& \text { Gef. " } 75.58,75.59, " 5.88,5.82 .
\end{array}
$$

Ihr Kupfersalz, $\left(\mathrm{C}_{16} \mathrm{H}_{13} \mathrm{O}_{2}\right)_{2} \mathrm{Cu}$, ist eine hellblave Fällung, sintert gegen $160^{\circ}$ und schmilzt bei $179^{\circ}$ vollständig zu einer klaren, gelben Flässigkeit.

$0.3135 \mathrm{~g}$ Sbst.: (bei $110^{\circ}$ getrocknet) $0.0432 \mathrm{~g} \mathrm{Cu} \mathrm{O}$.

$\left(\mathrm{C}_{16} \mathrm{H}_{13} \mathrm{O}_{3}\right)_{2} \mathrm{Cu}$. Ber. $\mathrm{CuO}$ 13.94. Gef. $\mathrm{CuO} 13.78$.

o-Xylalphtalid und alkoholisches Ammoniak,

geben, wenn man sie zwei Stunden im Rohr auf $100^{\circ}$ erhitzt, die Lösung concentrirt und mit Wasser versetzt, einen gelblich-weissen Krystallbrei, der aus verdünntem Alkohol in weissen Nadeln vom Schmp. $155^{\circ}$ anschiesst. Diese sind

$a-0-$ Metbyldesoxy-benzoïn-o-carbonsäureamid,

$\mathrm{NH}_{2} \cdot \mathrm{CO} . \mathrm{C}_{6} \mathrm{H}_{4} \cdot \mathrm{CO} \cdot \mathrm{CH}_{2} \cdot \mathrm{C}_{6} \mathrm{H}_{4} \cdot \mathrm{CH}_{3}$.

An Rohproduct ward eine Ausbeute von 81 pCt., an reiner Substanz von $65 \mathrm{pCt}$. der Theorie erzielt. In Wasser ist der Körper nicht, in Aether, Schwefelkohlenstoff und Ligroïn schwer, leichter in den anderen üblichen Mitteln löslich.

0.1622 g Sbst.: $0.4512 \mathrm{~g} \mathrm{CO}_{2}, 0.0918 \mathrm{~g} \mathrm{H}_{2} \mathrm{O}$.

$0.1732 \mathrm{~g}$ Sbst : $8.7 \mathrm{~cm} \mathrm{~N}(210,763.3 \mathrm{~mm})$.

$\mathrm{C}_{16} \mathrm{H}_{15} \mathrm{O}_{2} \mathrm{~N}$. Ber. C 75.84, H 5.94, N 5.54.

Gef. 》75.87, »6.29, 》5.76.

Kocht man das genannte Amid mit 10 Theilen Eisessig 10 Minuten lang und fügt dann in der Siedehitze Wasser bis zur Trübung hinzu, so scheidet sich beim Erkalten in gelben Nädelchen vom Schmp. $196-197^{\circ}$

$$
o \text {-Xylalphtalimidin, } \mathrm{C}_{6} \mathrm{H}_{4} \underbrace{\mathrm{C}: \mathrm{CH} . \mathrm{C}_{6} \mathrm{H}_{4} \cdot \mathrm{CH}_{3}}_{\mathrm{CO} . \mathrm{NH}},
$$

ab, welches in Wasser nicht, in Methyl-, Aethyl-, Amyl-Alkohol, Aether, Ligroïn und Schwefelkohlenstoff schwer, in warmem Benzol, Eisessig, Essigester und kaltem Chloroform sehr leicht löslich ist. Es lässt sich auch durch längeres Erhitzen mit alkoholischer Ammoniaklösung direct aus $o$-Xylalphtalid erbalten.

$0.1818 \mathrm{~g}$ Sbst.: $0.5446 \mathrm{~g} \mathrm{CO}_{2}, 0.0935 \mathrm{~g} \mathrm{H}_{2} \mathrm{O}$.

$0.1679 \mathrm{~g}$ Sbst.: $9.4 \mathrm{cem} \mathrm{N}\left(21.5^{0}, 745 \mathrm{~mm}\right)$.

$\mathrm{C}_{16} \mathrm{H}_{13} \mathrm{ON}$. Ber. C 81.70, H 5.54, N 5.96.

Gef. 》 $81.70, 》 5.71$, * 6.25 . 


\section{6}

Verbalten der a-o-Methyldesoxybenzoïn-o-carbonsäure.

1) Gegen Hydroxylamin. $1 \mathrm{~g}$ Säure wird in $24 \mathrm{ccm} 1 / 6$-Normalnatron gelöst, mit $4 \mathrm{ccm}$ Normalhydroxylaminlösung etwa 10 Minuten gekocht und dann mit $4 \mathrm{ccm}$ Normalsalzsäure versetzt, wobei eine teigige Fällung entsteht, die bei weiterem Kochen langsam erstarrt und aus Alkohol in glänzenden, prismatischen Blättchen vom Schmp. $138.5^{0}$ anschiesst.

Der Körper löst sich in Wasser und Ammoniak nicht, schwer in Aether und Ligroïn, leicht in warmem Methyl-, Aethyl-, AmylAlkohol, sehr leicht in Chloroform, Benzol, Eisessig, Essigester und Schwefelkohlenstoff und ist das

Oximidolacton der $u-0-$ Xylylphenylketoxim-o-carbon-

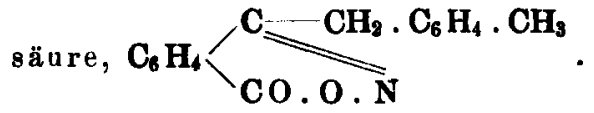

$$
\mathrm{C}_{16} \mathrm{H}_{13} \mathrm{O}_{2} \mathrm{~N} \text {. Ber. N 5.59. Gef. N } 5.97 \text {. }
$$

$0.1555 \mathrm{~g}$ Sbst.: $8: 2 \mathrm{ccm} \mathrm{N}\left(18^{0}, 746 \mathrm{~mm}\right)$.

2) Gegen Phenylhydrazin. a-o-Methyldesoxybenzoïn-o-carbonsäure und Phenylhydrazin ergeben, wenn man sie in Alkohol mit einigen Tropfen Essigsäure kurze Zeit auf dem Wasserbade erhitzt, sodann auf $50^{\circ}$ abkühlt und laues Wasser bis zur Trübung zusetzt, beim Abkühlen einen flockigen Niederschlag, der aus siedendem Alkohol in mikrokrystallinischen Nädelchen vom Schmp. $177^{\circ}$ anschiesst und aus

$$
\text { 2.4- } u \text {-o-Xylylphenylphtalazon, } \mathrm{C}_{6} \mathrm{H}_{4}<-\mathrm{CO}\left(\mathrm{C}_{8} \mathrm{H}_{9}\right): \stackrel{\mathrm{N}}{\mathbf{N}} . \mathrm{C}_{6} \mathrm{H}_{3},
$$

besteht. Es zeigt ähnliche Löslichkeit wie die vorige Verbindung.

$$
\mathrm{C}_{92} \mathrm{H}_{18} \mathrm{~N}_{2} \mathrm{O} \text {. Ber. N 8.61. Gef. N } 8.96 \text {. }
$$

$0.1788 \mathrm{~g}$ Sbst.: $14.5 \mathrm{~cm} \mathrm{~N}\left(21^{0}, 738 \mathrm{~mm}\right)$.

3) Gegen Natriumamalgam. $30 \mathrm{~g}$ o-Xylalphtalid werden durch Alkali zu Methyldesoxybenzoïn $o$-carbonsäure gelöst (s. o.), die Lösung mit etwa $400 \mathrm{ccm}$ Wasser verdünnt und unter Kühlen mit $600 \mathrm{~g}$ 21/2-procentigem Natriumamalgam durchgeschüttelt. In der Lösung (A) ist nunmehr eine im freien Zustande wenig beständige Oxysäure $=\mathrm{CO}_{2} \mathrm{H} \cdot \mathrm{C}_{6} \mathrm{H}_{4} \cdot \mathrm{CH}(\mathrm{OH}) \cdot \mathrm{CH}_{2} \cdot \mathrm{C}_{7} \mathrm{H}_{7}$ ( $(x-o$-Methyltoluylenhydrat-o-carbonsäure) ') vorhanden, welche sich durch Salzsäure teigig abscheidet, sich nur theilweise in Ammoniak wieder löst und ihre Ammoniaklöslichkeit beim Erwärmen verliert.

1) wa zum Unterschied von der weiter unten beschriebenen "; $;$-Säure genannt. 
Aus siedendem Alkohol umkrystallisirt, liefert das Product wasserbelle Prismen vom Schmp. $87^{\circ}$, welche das der Oxysäure entsprechende Lacton, d. h.

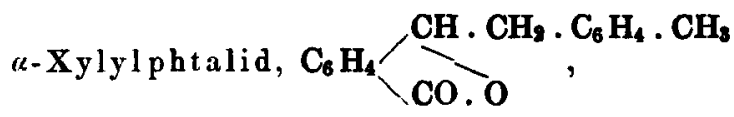

darstellen.

Es ist in den üblichen Lösungsmitteln, Wasser und Ligroïn ausgenommen, leicht löslich.

0.1735 g Sbst.: $0.5125 \mathrm{~g} \mathrm{CO}_{2}, 0.0961 \mathrm{~g} \mathrm{H}_{2} \mathrm{O}$.

$$
\begin{aligned}
& \mathrm{C}_{16} \mathrm{H}_{14} \mathrm{O}_{2} \text {. Ber. C 80.63, H } 5.89 \text {. } \\
& \text { Gef. 80.56, } 6.15 \text {. }
\end{aligned}
$$

Eine krystallographische Untersuchung, welche ich ebenso wio die folgenden, Hrn. Gymnasial-Oberlehrer C. Täuber, Breslau, verdanke, ergab:

»Krystallsystem: monosymmetrisch.

Die farblosen durchsichtigen Krystalle sind gestreckt nach der Symmetrieaxe und tafelig nach einer zur Symmetrieebene senkrechten Fläche ausgebildet. Ausserdem sind sie begrenzt von zwei anderen, zur Symmetrieebene senkrechten Flächenpaaren. Die Tafelfäche als Querfläche $\alpha=(100)$ und eine der andern, zu dieser unter einem Winkel von $66^{1} /^{\circ}$ geneigte, als Basis $\mathrm{c}=(001)$ genommen, also die Axenschiefe $\beta=1131 / 2^{0}$, ergiebt die dritte zur Symmetrieebene senkrechte Fläche als positives Hemidoma. Dieses als primäres $\mathbf{x}=(\overline{101})$ genommen, zur Querfläche unter $67^{\circ}$ und zar Basis unter 46 $1 / 8^{0}$ geneigt, ergiebt das Axenverhältniss:

$$
\mathrm{a}: \mathrm{c}=1: 0.7880 \text {. }
$$

Seitlich waren die Krystalle sämmtlich verbrochen und liessen keine prismatischen Endfächen beobachten. Eine Spaltbarkeit wurde nicht wahrgenommen. Die Ebene der optischen Axen steht senkrecht zur Symmetrieebene. Durch die Tafelfäche $\alpha=(100)$ treten in Cedernholzöl (von den Brechungsquotienten

$$
\begin{aligned}
& \mathrm{n}=1.4966 \text { für } \mathrm{Li}-\text { Licht } \\
& \mathrm{n}=1.4993 \text { für } \mathrm{Na}-\text { Licht } \\
& \mathrm{n}=1.5020 \text { für } \mathrm{Tl} \text { - Licht) }
\end{aligned}
$$

unter etwa $52^{\circ}$ gegen die Normale zur Querfläche geneigt, die optischen Axen mit einer scheinbaren Apertur von etwa $60^{\circ}$ für Natriumlicht aus.

In welchem Sinne die Axenebene zur Querfäche geneigt ist, war wegen der mangelhaften geometrischen Orientirung der Krystalle nicht zu entscheiden, indem die $Z$ ugehörigkeit zum monosymmetrischen (nicht rhombischen) Krystallsystem nicht mit Sicherheit aus den Winkelmessungen, sondern lediglich aus dem optischen Verhalten (aus 


\section{8}

diesem aber mit Bestimmtheit) geschlossen werden konnte. Wegen der sehr starken Doppelbrechung war der optische Charakter der I. Mittellinie nicht mit Sicherheit zu bestimmen.《

Ausser dem vorgenannten Lacton kann man aus der unbeständigen Oxysäure durch Wasserabspaltung auch eine ungesättigte Säure gewinnen und zwar wie folgt: Auf dem Wasserbade eingedampft, ergab die alkalische Lösung (A) einen Syrup, der auf der Kalilauge schwamm; letztere wurde mit der Pipette abgesogen und der Syrup im Luftbade fünf Stunden lang auf etwa $200^{\circ}$ erhitzt. Die erstarrte Schmelze löste man in Wasser und säuerte noch beiss mit verdünnter Salzsäure an. Die hierbei entstehende, weisse Fällung ergab, aus siedendem Alkohol umkrystallisirt, schneeweisse, zu Büscheln und Sternchen vereinigte Nadeln einer ungezättigten Säure (=24 g) rom Schmp. $169^{\circ}$, die durch Wasserabspaltung aus der obigen Oxysäure entstanden und als

$$
\begin{aligned}
& 0-\mathrm{Methylstilben-o-carbonsäure,} \\
& \mathrm{CO}_{2} \mathrm{H} . \mathrm{C}_{6} \mathrm{H}_{4} \cdot \mathrm{CH}: \mathrm{CH} . \mathrm{C}_{6} \mathrm{H}_{4} \cdot \mathrm{CH}_{3},
\end{aligned}
$$

zu bezeichnen ist. Sie löst sich schwer in Wasser sowie Ligroïn, leichter in warmem Methyl-, Aethyl-, Amyl-Alkohol, Aether, Benzol und Eisessig, ziemlich leicht in Schwefelkohlenstoff, Chloroform und Essigester, leicht in Ammoniak.

$$
\begin{aligned}
& 0.1618 \text { g Sbst.: } 0.4783 \mathrm{~g} \mathrm{CO}_{2}, 0.0912 \mathrm{~g} \mathrm{H}_{2} \mathrm{O} \text {. } \\
& \mathrm{C}_{16} \mathrm{~B}_{14} \mathrm{O}_{2} \text {. } \begin{array}{l}
\text { Ber. C 80.63, N } 5.89 . \\
\text { Gef. 》 } 80.62, » 6.26 .
\end{array}
\end{aligned}
$$

Ihr grünes $\mathrm{Kupfersalz},\left(\mathrm{C}_{16} \mathrm{H}_{13} \mathrm{O}_{2}\right)_{2} \mathrm{Cu}$, ist in Wasser and Alkohol nicht, in Aether mit grüner Farbe löslich -nd schiesst aus Aether in saftgrünen Säulen an, die sich gegen $120^{\circ}$ verfärben und bei etwa $150^{\circ}$ unter Aufschäumen und Dunkelfärbung schmelzen.

$0.3262 \mathrm{~g}$ Sbst.: (vacuumtrocken) $0.0494 \mathrm{~g} \mathrm{CuO}$.

$$
\left(\mathrm{C}_{16} \mathrm{H}_{13} \mathrm{O}_{2}\right)_{2} \mathrm{Cu} \text {. Ber. } \mathrm{CuO} \text { 14.83. Gef. } \mathrm{CuO} \text { 15.14. }
$$

Verhalten der o-Methylstilben-o-carbonsäure.

1. Gegen Wasserstoff. $1 \mathrm{~g}$ o-Methylstilben-o-carbonsäure wird mit Natronlauge gelöst, auf etwa $30 \mathrm{ccm}$ mit Wasser verdünnt und mit $25 \mathrm{~g} \mathrm{2} / 2$-procentigem Natriumamalgam versetzt. Beim Stehen über Nacht hatte sich ein dichter Krystallbrei langer Nadeln, das Natriumsalz der erwarteten

o-Metbyldibenzyl-o-carbonsäure,

$\mathrm{CO}_{2} \mathrm{H} \cdot \mathrm{C}_{6} \mathrm{H}_{4} \cdot \mathrm{CH}_{2}, \mathrm{CH}_{2} \cdot \mathrm{C}_{6} \mathrm{H}_{4} \cdot \mathrm{CH}_{3}$

ausgeschieden.

Die freigemachte neue Säure krystallisirt aus etwa 60-procentigem Alkohol in Blättchen and Prismen, die bei $120^{\circ}$ erweichen, bei $123^{\circ}$ 
schmelzen and wenig in Wasser und in Ligroïn, leicht in den anderen üblichen Lösungsmitteln sich lösen.

0.1646 g Sbst.: $0.4809 \mathrm{~g} \mathrm{CO}_{2}, 0.1020 \mathrm{~g} \mathrm{H}_{2} \mathrm{O}$.

$$
\begin{aligned}
& \mathrm{C}_{16} \mathrm{H}_{16} \mathrm{O}_{2} \text {. Ber. C 79.95, H 6.68. } \\
& \text { Gef. \ 79.68, : } 6.88 \text {. }
\end{aligned}
$$

2. Gegen Brom. $3 \mathrm{~g} o$-Methylstilben-o-carbonsäure werden in $40 \mathrm{ccm}$ heissem Eisessig allmählich mit einer Lösung von $2 \mathrm{~g}$ Brom in $20 \mathrm{ccm}$ Essigsäure versetzt, dessen Farbe unter Entwickelung von Bromwasserstoff sofort verschwindet. Nachdem man die Lösung concentrirt bat, fällt man mit heissem Wasser eine zähflüssige Masse, die durch Umkrystallisiren aus absolutem Alkohol weisse, durchsichtige Nadeln vom Schmp. 101-1020 darstellt. In Wasser ist der Körper nicht, in Ligroïn und Aether schwer, in warmem Methyl-, Amyl-Alkohol und kaltem Benzol, Eisessig, Chloroform, Essigester und Schwefelkohlenstoff sehr leicht löslich.

Der bromhaltige Körper hat die Formel $\mathrm{C}_{16} \mathrm{H}_{13} \mathrm{O}_{2} \mathrm{Br}$.

0.1835 g Sbst.: $0.1084 \mathrm{~g} \mathrm{AgBr}$.

$$
\mathrm{C}_{16} \mathrm{H}_{13} \mathrm{O}_{2} \text { Br. Ber. 25.24. Gef. 25.14. }
$$

$\mathrm{Da}$ er offenbar aus dem intermediär gebildeten Dibromid $\mathrm{CO}_{2} \mathrm{H}$. $\mathrm{C}_{6} \mathrm{H}_{4} \cdot \mathrm{CH} \mathrm{Br} . \mathrm{CHBr} \cdot \mathrm{C}_{7} \mathrm{H}_{7}$ dureh Austritt von $\mathrm{HBr}$ hervorgegangen und unlöslich in Ammoniak, also keine Säure ist, so muss ihm eine der Formeln

I.

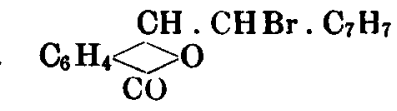

II. $\mathrm{C}_{6} \mathrm{H}_{4}<\underset{\mathrm{CO}-\mathrm{O}}{\mathrm{CH} \mathrm{Br}}>\mathrm{CH} . \mathrm{C}_{7} \mathrm{H}_{7}$ zukommen.

Durch Abspaltung von noch $1 \mathrm{Mol}$. $\mathrm{HBr}$ mus, sich, je nachdem I oder II zutrifft,

Ia.

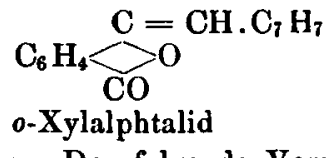

oder
IIa. $\mathrm{C}_{6} \mathrm{H}_{4}<\begin{aligned} & \mathrm{CH}=\underset{\mathrm{CO}}{\mathrm{C}} \cdot \dot{\mathrm{O}} \\ & \mathrm{C}_{7} \mathrm{H}_{7}\end{aligned}$ $o$-Tolylisocumarin

ergeben. Der folgende $V$ ersuch hat für die letztere Möglichkeit entschieden, sodass also der Körper $\mathrm{C}_{16} \mathrm{H}_{13} \mathrm{O}, \mathrm{Br}$ gemäss der Formel II als 1-Brom-3-o-tolyl-dibydroisocumarin zu bezeichnen ist.

Zur Abspaltung von $\mathrm{HBr}$ aus $\mathrm{C}_{16} \mathrm{H}_{13} \mathrm{O}_{2} \mathrm{Br}$ braucht man letzteres nicht erst zu isoliren, sondern kann unter erheblicher Verbesserung der Ausbeute direct in folgender Weise verfahren.

$24 \mathrm{~g} \mathrm{o-Methylstilben-o-carbonsäure} \mathrm{in} 320 \mathrm{ccm}$ heissem Eisessig werden mit $16 \mathrm{~g}$ Brom in $50 \mathrm{ccm}$ Eisessig versetzt, die Lösung über freier Flamme eingeengt, dann im Vacuum bei $100^{\circ}$ von Essigsäure befreit und schliesslich unter $12 \mathrm{~mm}$ Druck destillirt, wobei das Product $(19 \mathrm{~g}) \mathrm{zwischen} 220-240^{\circ}$ übergeht. Der zur Analyse bestimmte Antheil wurde aus seiner Lösung in Scbwefelkohlenstoff und Ligroïn unter Eiskühlung in schönen, gelben Krystallen rom Schmp. 102.5 
erhalten. Er ist in Wasser nicht, in Ligroön schwer, in warmem Methyl-, Aethyl-, Amyl-Alkohol, Aether, Eisessig und Essigester, sowie kaltem Schwefelkohlenstoff, Benzol und Chloroform leicht löslich und besteht aus

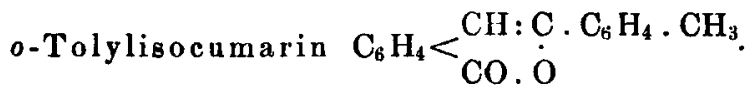

Das isomere $o$-Xylalphtalid schmilzt bei $136.5^{\circ}$, also $34^{0}$ höher als o-Tolylisocumarin.

Die nach den vorbeschriebenen Reactionen durchzuführende Umwandlung des $o$-Xylalphtalids in $o$-Tolylisocumarin lieferte $64 \mathrm{pCt}$. Ausbeute.

Krystallographische Angaben über o-Tolylisocumarin:

Krystallsystem: rhombisch.

Axenverhältniss : $\mathrm{a}: \mathrm{b}: \mathrm{c}=0.75264: 1: 0.54994$.

$$
\begin{gathered}
\text { Beobachtete Formen: } \\
\mathrm{m}=(110), \mathrm{d}=(011) . \\
\text { Winkeltabelle: }
\end{gathered}
$$

$\begin{array}{rlrl} & & \text { Berech net: } & \text { Beobachtet: } \\ \mathbf{m}: \mathbf{m}=(110):(1 \overline{1} 0) & - & { }^{\prime} 73^{0} 5 i^{\prime} \\ \mathrm{d}: \mathbf{d}=(011):(0 \overline{1} 1) & - & { }^{*} 57^{0} 37^{\prime} \\ \mathbf{m}: \mathbf{d}=(110):(011) & 73^{\circ} 9^{\prime} & 73^{\circ} 8^{\prime}\end{array}$

Die blassgelben, wenig durchsichtigen Krystalle sind mehr oder weniger säulenförmig nach der Verticalen ausgebildet, zuweilen etwas verkürzt, sodass der Habitus ein octaëdrisches Ansehen gewinnt.

Fine Spaltbarkeit warde nicht beobachtet.

Die optische Axenebene ist die Basis. Durch jede Prismenfläche tritt eine Axe mit sehr starker Dispersion aus.

\section{Verhalten des $o$-Tolylisocumarins.}

1. Gegen Kali. Erwärmt man $1 \mathrm{~g} \mathrm{3-o-Tolylisocumarin} \mathrm{mit} 3 \mathrm{ccm}$ concentrirter Kalilauge, so verwandelt es sich in einen grünlichen Syrup, der von der Kalilauge getrennt und mit Wasser aufgenommen wird. Aus dieser Lösung fällt Salszäure

$$
\begin{aligned}
& \not \beta-o \text { - Methyldesoxybenzoïn-o-carbonsäure }{ }^{1} \text { ), } \\
& \mathrm{HO}_{2} \mathrm{C} \cdot \mathrm{C}_{6} \mathrm{H}_{4} \cdot \mathrm{CH}_{2} \cdot \mathrm{CO} \cdot \mathrm{C}_{6} \mathrm{H}_{4} \cdot \mathrm{CH}_{3} \text {, }
\end{aligned}
$$

die aus verdünntem Alkohol in farblosen Nadeln vom Schmp. $139^{\circ}$ anschiesst. In den üblichen Lösungsmitteln ist sie leicht, in Ligroïn schwerer und in Wasser nicht löslich. Durch Schmelzen geht sie in 3-o-Tolylisocumariu zurück.

0.1659 g Sbst.: $0.4588 \mathrm{~g} \mathrm{CO}_{2}, 0.0863 \mathrm{~g} \mathrm{H}_{2} \mathrm{O}$.

$$
\begin{aligned}
& \mathrm{C}_{66} \mathrm{H}_{14} \mathrm{O}_{3} \text {. Ber. C 75.59, H 5.52. } \\
& \text { Gef. 》 75.42, 》5.78. }
\end{aligned}
$$

1) Ueber die isomere a-Sanre s. w. oben. 
Reducirt man diese Säure in alkalischer Lösung mit Natriumamalgam, so fällt auf nachherigen Zusatz von Salzsäure ein Niederschlag aus, der im Gegensatz zu der entsprechenden $\alpha$-o-Metbyltoluylenhydrat-o-curbonsäure (s. o.) selbst nach dem Umkrystallisiren aus siedendem Alkohol noch saure Eigenschaften beibehält und aus

$$
\begin{gathered}
\beta-o-\text { Methyl-toluylenhydrat-o-carbonsäure'), } \\
\mathrm{HO}_{2} \mathrm{C} \cdot \mathrm{C}_{6} \mathrm{H}_{4} \cdot \mathrm{CH}_{2}(\mathrm{OH}) . \mathrm{CH}(\mathrm{OH}) \cdot \mathrm{C}_{6} \mathrm{H}_{4} \cdot \mathrm{CH}_{3},
\end{gathered}
$$

besteht. Sie stellt feine, weisse Nadeln dar, die bei 137", langsam erhitzt schon etwas tiefer, unter lebhaftem Aufschäumen schmelzen. In den gewöbnlichen Lösungsmitteln ist sie leicbt löslich, schwerer in Ligroïn.

$$
\begin{aligned}
& 0.1786 \text { g Sbst.: } 0.4891 \mathrm{~g} \mathrm{CO}_{2}, 0.1068 \mathrm{~g} \mathrm{H}_{2} \mathrm{O} \text {. } \\
& \mathrm{C}_{16} \mathrm{H}_{16} \mathrm{O}_{3} \text {. Ber. C 74.95, H 6.27. } \\
& \text { Gef. 》74.69, } 76.64 \text {. }
\end{aligned}
$$

Es stand zu erwarten, dass im gleichen Sinne, wie daq $\alpha-0-$ methyltoluylenhydrat-o-carbonsaure Kalium auch das Kaliumsalz der $\beta$-Verbindung durch Erhitzen Wasser abspalten, d. b. die o-Methylstilben-o-carbousäure vom Schmp. $169^{\circ}$ liefern würde. Diese Vermuthung fand ich bestätigt.

Es gelten also die Gleichungen

$$
\left.\begin{array}{l}
\mathrm{C}_{6} \mathrm{H}_{4}<\underset{\mathrm{CO}}{\mathrm{CH}(\mathrm{OH})} \cdot \mathrm{CH}_{2} \cdot \mathrm{C}_{7} \mathrm{H}_{7} \\
\mathrm{C}_{6} \mathrm{H}_{4}<{ }_{\mathrm{CO}_{2} \mathrm{~K}}^{\mathrm{CH}_{2} \cdot \mathrm{CH}(\mathrm{OH}) \cdot \mathrm{C}_{7} \mathrm{H}_{7}}
\end{array}\right\}=\mathrm{C}_{6} \mathrm{H}_{4}<\underset{\mathrm{COOK}}{\mathrm{CH}: \mathrm{CH}_{7} \mathrm{C}_{7} \mathrm{H}_{7}}+\mathrm{H}_{2} \mathrm{O} .
$$

Dagegen führt die Wasserabspaltung aus der freien $\beta$-Säure zu einem von dem o-Xylylphtalid (Schmp. 87") verschiedenen Product.

B.o-Methyltoluylenhydrat-o-carbonsäure giebt nämlich bei $150^{\circ}$ unter Aufschäumen eine Schmelze, die, aus siedendem Alkohol umkrystallisirt, schneeweisse Nadeln vom Schmp. 117" liefert. Sie sind

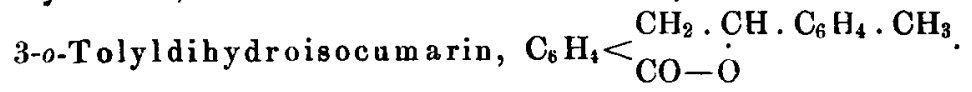

Das Lacton ist in Wasser und Ligroïn schwierig, leicht in Alkohol etc. löslich. Es bildet sich auch allmählich beim Kochen eiver ammoniakalischen Lösung der $\beta$-o-Säure.

$$
\begin{aligned}
& 0.1491 \mathrm{~g} \text { Sbst: } 0.0803 \mathrm{~g} \mathrm{H}_{2} \mathrm{O}, 0.4395 \mathrm{~g} \mathrm{CO} . \\
& \mathrm{C}_{16} \mathrm{H}_{14} \mathrm{O}_{2} . \text { Ber. C } 80.63 \text {, H } 5.89 . \\
& \text { Gef. " } 80.39, " 5.98 .
\end{aligned}
$$

2. Gegen Ammoniak. $100 \mathrm{ccm}$ alkoholisches Ammoniak und $10 \mathrm{~g}$ o-Tolylisocumariu liefern, wenn man sie 12 Stunden in Rohr auf 1000 erhitzt und daun die Lösung einengt, $7.8 \mathrm{~g}$

$$
\text { 3-o-Tolylisocarbostyril, } \mathrm{C}_{6} \mathrm{H}_{4}<\mathrm{CO} \text {. } \mathrm{NH}
$$

Es tritt in farblosen, mikroskopischen Tăfelchen vom Schmelzpunkt $179^{\circ}$ auf, löst sich nicht in Wasser, schwer in Ligroïn, leichter in 


\section{2}

warmem Methyl-, Aethyl-, Amyl-Alkohol, Aether and kaltem Benzol, Eisessig, Chloroform, Essigester und Schwefelkohlenstoff.

$0.1722 \mathrm{~g}$ Sbst.: $0.5165 \mathrm{~g} \mathrm{CO}_{2}, 0.0913 \mathrm{~g} \mathrm{H}_{2} \mathrm{O}$.

0.1851 g Sbst.: $9.7 \mathrm{ccm} \mathrm{N}\left(17^{0}, 740.5 \mathrm{~mm}\right)$.

$$
\begin{aligned}
& \mathrm{C}_{16} \mathrm{H}_{13} \mathrm{ON} \text {. Ber. C 81.70, H 5.54, N } 5.96 \text {. } \\
& \text { Gef. 》 81.78, 》5.89, 》5.94. }
\end{aligned}
$$

Phosphoroxychlorid $(8 \mathrm{ccm})$ und o-Tolylisocumarin $(4 \mathrm{~g})$ geben, eine halbe Stunde auf dem Wasserbade erhitzt, eine violetrothe Lösung, die man in $80 \mathrm{ccm}$ Eiswasser langsam eingiesst. Die allmählich erstarrte Fällung wird getrocknet und im Vacuum destillirt. Das farblose, $\mathrm{zwischen} 200$ und $225^{\circ}$ anfgefangene Destillat $\left(4^{1} / 4 \mathrm{~g}\right)$ gesteht allmählich zu einer weissen, festen Masse, die aus siedendem Alkohol in wasserhellen Krystallen vom Schmp. $67^{\circ}$ anschiesst. Sie lösen sich nicht in Wasser, leicht in warmem Methyl-, Aethyl-, Amyl-Alkohol, kaltem Aether, Benzol, Eisessig, Chloroform, Essigester und Schwefelkohlenstoff und werden aus ihrer Lösung in concentrirter Salzsäure durch Wasser wieder gefällt.

$0.1895 \mathrm{~g}$ Sbst.: $0.1059 \mathrm{~g} \mathrm{AgCl}$.

$\mathrm{C}_{16} \mathrm{H}_{12} \mathrm{NCl}$. Ber. $\mathrm{Cl}$ 13.98. Gef. Cl 13.82.

Der Körper ist demnach

1-Chlor-3-o-Tolylisochinolin, $\mathrm{C}_{6} \mathrm{H}_{4}<\begin{aligned} & \mathrm{CH}: \underset{\mathrm{CCl}: \dot{\mathrm{N}}}{\mathrm{C}} \cdot \mathrm{C}_{6} \mathrm{H}_{4} \cdot \mathrm{CH}_{3} \\ & \text { 年 }\end{aligned}$

Eine Krystallbestimmung ergab:

Krystallsystem: monosymmetrisch.

$\mathrm{a}: \mathrm{b}: \mathrm{c}=1.35858: 1: 1.05065$.

$$
\beta=119045^{\prime}
$$

\begin{tabular}{|c|c|c|}
\hline$m: m=(1] 0):(1 \overline{1} 0)$ & Berechnet & $\begin{array}{l}\text { Beobachtet } \\
{ }^{*} 99^{\circ} 25^{\prime}\end{array}$ \\
\hline $\mathrm{m}: \mathbf{a}=(110):(100)$ & $49^{0} 42^{1} / \mathbf{2}^{\prime}$ & $49^{0} 421 / 2^{\prime}$ \\
\hline $\mathbf{m}: c=(110):(001)$ & - & $* 71^{0} 177^{\prime}$ \\
\hline$c: a=(001):(100)$ & $60^{0} 15^{\prime}$ & $60^{\circ}$ appr. \\
\hline$a: x=(100):(20 \overline{1})$ & $30^{\circ} 34^{1 / 2}$ & $39^{3} / 4^{0}$ appr. \\
\hline $\mathrm{x}: \mathrm{c}=(\overline{2} 01):(001)$ & $80^{0} 10^{1} / \mathrm{a}^{\prime}$ & $80^{1} / 4^{0}$ appr. \\
\hline $\mathrm{c}: \mathrm{o}=(001):(111)$ & - & ${ }^{*} 6102 \vec{\prime}$ \\
\hline $0: \mathrm{m}=(11 \overline{1}):(110)$ & $47^{0} 16^{\prime}$ & $47^{\circ} \quad 16^{\prime}$ \\
\hline $\mathrm{m}: \mathrm{o}=(110):(\overline{1} 11)$ & $66^{0} 23^{\prime}$ & $66^{0} 28^{\prime}$ \\
\hline $0: x=(\overline{1} 11):(\overline{2} 01)$ & $53^{0} 31^{\prime}$ & $53^{0} 27^{\prime}$ \\
\hline $\mathrm{x}: \mathrm{m}=(20 \overline{\mathrm{l}}):(110)$ & $60^{\circ} \quad 6^{\prime}$ & $60^{\circ} \quad 5^{\prime}$ \\
\hline
\end{tabular}

Beobachtete Formen: 


\section{3}

Die farblosen Krystalle, in den vorliegenden Exemplaren nur von winziger Grösse, sind von annāhernd rhomboëdrischem Habitus durch ungefāhr gleiche Ausdebnung von $\mathrm{cm}$ einerseits und $\mathrm{a} o$ andererseits; $\mathrm{cm}$ herrschend. Eine Spaltbarkeit wurde nicht beobachtet.

Eine vähere optische Untersuchung war an dem vorliegenden Material nicht ausführbar, zumal eine solche nicht durch eine zufällig günstige Lage der Ebene der optischen Axen erleichtert wurde.

Reduction der Chlorbase. $3 \mathrm{~g}$-Chlor- $\beta$-o-Tolylisochinolin und $1.2 \mathrm{~g}$ rother Phosphor werden mit $12 \mathrm{ccm}$ Jodwasserstoffsäure vom Sdp. $127^{\circ}$ drei Stunden gekocht. Nach dem Erkalten gesteht die Flüssigkeit zu einer gelben krystallinischeu Masse, die man in heissem Wasser löst, heiss vom Phosphor abfiltrirt, worauf beim Erkalten das Jodhydrat des

$$
\text { 3-o-Tolylisochinolins, } \mathrm{C}_{6} \mathrm{H}_{4}<\begin{aligned}
& \mathrm{CH}: \underset{\mathrm{CH}}{\mathrm{C}} \cdot \mathrm{C}_{6} \mathrm{H}_{4} \cdot \mathrm{CH}_{3} \\
& \text {, }
\end{aligned}
$$

anscbiesst ( $85 \mathrm{pCt}$. der Theorie).

Die daraus mit Kalilauge abgeschiedene Base ( $1.8 \mathrm{~g}$ ) krystallisirt aus eiskaltem Ligroïn in weissen körnigen Krystallen vom Schmp. 78-790 und ist leicht löslich in den üblichen Mitteln.

0.1933 g Sbst.: $0.1057 \mathrm{~g} \mathrm{CO}_{2}, 0.6199 \mathrm{~g} \mathrm{~B}_{2} \mathrm{O}$.

0.1717 g Sbst.: $9.8 \mathrm{ccm} \mathrm{N}\left(17^{\circ}, 753.5 \mathrm{~mm}\right)$.

$$
\begin{aligned}
& \mathrm{C}_{16} \mathrm{H}_{13} \mathrm{~N} \text {. Ber. C 87.64, H 5.95, N 6.41. } \\
& \text { Gef. 》 87.46, 》6.08, 》6.56. }
\end{aligned}
$$

Das Chlorhydrat stellt feine Nädelchen dar, die bei $218^{\circ}$ erweichen und bei $221^{\circ}$ schmelzen, das Bromhydrat warzenförmig angeordnete Nädelcben, die gegen $200^{\circ}$ erweichen und unter Bräunung bei $235^{n}$ schmelzen, das Jodhydrat goldgelbe Nadeln, die gegen $190^{\circ}$ unter Zersetzung und Dunkelfürbung schmelzen, das Pikrat Stäbchen vom Schmp. ca. $150^{\circ}$, das Sulfat Nadelbüschel, bei $200^{\circ}$ erweichend, gegen $212^{\circ}$ schmelzend. Das Chlorplatinat, $\left(\mathrm{C}_{16} \mathrm{H}_{14} \mathrm{~N}\right)_{8} \mathrm{PtCl}_{6}$ $+2 \mathrm{H}_{2} \mathrm{O}$, schiesst aus Alkohol in mikroskopischen goldorangegelben Täfelchen an, die bei $210^{\circ}$ unter Aufschüumen schmelzen.

$0.0888 \mathrm{~g}$ vacuumtrockne Substanz gaben bei $105^{\circ} 0.0037 \mathrm{~g} \mathrm{H}_{2} \mathrm{O}$ und verglüht $0.0194 \mathrm{~g} \mathrm{Pt}$.

$$
\begin{aligned}
& \left(\mathrm{C}_{16} \mathrm{H}_{14} \mathrm{~N}_{2} \mathrm{PtCl}_{6}+2 \mathrm{H}_{2} \mathrm{O} .\right. \\
& \begin{array}{l}
\text { Ber. } \\
\text { Gef. }
\end{array} \mathrm{H}_{2} \mathrm{O} \text { 4.08, } \\
&
\end{aligned}
$$

